12,10

\title{
Структурные и электронные свойства нового соединения $\mathrm{SrOs}_{2} \mathrm{O}_{6}$ *
}

\author{
() П.А. Агзамова ${ }^{1,2}$, С.В. Стрельцов ${ }^{1,2}$ \\ ${ }^{1}$ Институт фризики металлов им. М.Н. Михеева УрО РАН, \\ Екатеринбург, Россия \\ ${ }^{2}$ Уральский федеральный университет им. Б.Н. Ельцина, \\ Екатеринбург, Россия \\ E-mail: polly@imp.uran.ru \\ Поступила в Редакцию 8 июля 2021 г. \\ В окончательной редакции 13 июля 2021 г. \\ Принята к публикации 16 июля 2021 г.
}

\begin{abstract}
Теоретически показана возможность существования нового соединения $\mathrm{SrOs}_{2} \mathrm{O}_{6}$, кристаллическая структура которого состоит из слоев октаэдров $\mathrm{OsO}_{6}$, связанных через общие ребра и формирующие решетку типа пчелиные соты. Исследованы структурные и электронные свойства $\mathrm{SrOs}_{2} \mathrm{O}_{6}$ с помощью расчетов в рамках теории функционала плотности. Показано, что энергетически выгодной для исследуемого соединения является антиферромагнитная структура.
\end{abstract}

Ключевые слова: $a b$ initio расчеты, низкоразмерный магнетизм, зонная структура.

DOI: 10.21883/FTT.2022.01.51844.27s

\section{1. Введение}

Слоистые системы на основе переходных металлов, имеющие решетку типа пчелиные соты, активно исследуются в последнее время. Некоторые из них, например, $\alpha-\mathrm{RuCl}_{3}, \quad \mathrm{Na}_{2} \mathrm{IrO}_{3}$ или $\mathrm{Li}_{2} \mathrm{IrO}_{3}$ представляют интерес из-за возможности реализации спин-жидкостного состояния и являются примерами систем, магнитные свойства которых описываются моделью Китаева-Гейзенберга [1-3]. В других соединениях наблюдается состояние типа жидкости валентных связей [4-6].

Важной особенностью таких систем в случае, когда переходный металл имеет незаполненную $t_{2 g}$-оболочку, является существование квазимолекулярных орбиталей, центрированных на шестиугольниках (гексагонах). Квазимолекулярные орбитали дают особый электронный спектр, а зонные состояния могут быть классифицированы с помощью неприводимых представлений группы $D_{6 h}$ (так же как и в случае молекулы бензола) [7,8]. Однако, такие факторы как спин-орбитальное взаимодействие, число валентных электронов, тенденция к димеризации, различные искажения кристаллической решетки влияют на то, реализуется состояние с квазимолекулярными орбиталями или нет [9]. В качестве материалов, в которых формирование квазимолекулярных орбиталей может оказывать существенное влияние на физические свойства системы, можно выделить такие оксиды Ru как $\mathrm{SrRu}_{2} \mathrm{O}_{6}[10-14]$ и $\mathrm{AgRuO}_{3}[15,16]$. Важно отметить, что в этих соединениях ион $\mathrm{Ru}$ имеет валентность 5+, что соответствует трем электронам на $t_{2 g}$-оболочке данного иона или шести $d$-электронам на элементар-

* Доклад на XXI Всероссийской школе-семинаре по проблемам физики конденсированного состояния вещества (СПФКС-21), Екатеринбург, 18-25 марта 2021 г. ную ячейку. В этом случае квазимолекулярные орбитали симметрии $B_{1 u}$ и $E_{2 g}$ оказываются полностью заполненными и в зонном спектре открывается щель, см. рис. 1 и [9]. При этом важно, что расщепления в данном спектре определяются величинами интегралов перескока $t_{p d}$ с узла на узел через $p$-орбитали лиганда [7]. В этой связи интересным является вопрос о том, каким образом изменится картина квазимолекулярных орбиталей, электронная структура в целом и физические свойства системы при замене $4 d$-иона переходного металла на $5 d$. Ведь в этом случае за счет увеличения главного квантового числа будет увеличиваться протяженность $d$-орбиталей, а значит и расти величина $t_{p d}[17,18]$.

В настоящей работе выполнено моделирование структурных и электронных свойств соединения $\mathrm{SrOs}_{2} \mathrm{O}_{6}$ на основе $5 d$-металла Os. Путем вычисления энтальпии образования показано, что такой материал является стабильным. Изучены кристаллическая структура и зонный спектр данной системы.

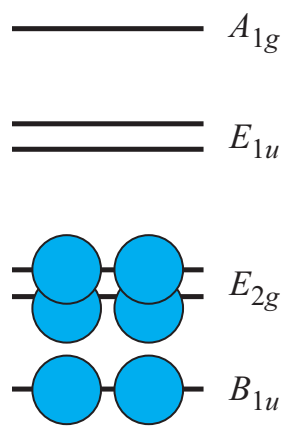

Рис. 1. Распределение электронов по квазимолекулярным орбиталям в случае электронной конфигурации $d^{3}$. 


\section{2. Методика расчета}

В представленной работе моделирование структурных и электронных свойств соединения $\mathrm{SrOs}_{2} \mathrm{O}_{6}$ было проведено в рамках методов теории функционала плотности (density functional theory, DFT) с использованием пакета VASP (Vienna Ab-initio Simulation Package) [19]. Вычисления проводились в рамках приближения обобщенного градиента (general gradient approximation, GGA) в формулировке PBE (Perdew-Burke-Ernzerhof) [20]. В расчетах энергия обрезания плоских волн $E_{\text {cutoff }}$ была взята равной $520 \mathrm{eV}$. Число $k$-точек в первой зоне Бриллюэна было выбрано в виде сетки $5 \times 5 \times 5$, построенной с помощью схемы Монхорста-Пака [21] для всех исследуемых структур. Расчеты были выполнены с использованием приближения проекционных присоединенных волн (projected augmented wave) - PAWпотенциалов [22] $\mathrm{Sr}(4 s 4 p 5 s)$, Os $(5 p 6 s 5 d)$ и О $\left(s^{2} p^{4}\right)$. Исходные параметры кристаллической структуры были взяты для $\mathrm{SrO}_{2}$ из [23] и для $\mathrm{OsO}_{2}$ из [24].

\section{3. Результаты и обсуждение}

Для вычисления энтальпии образования соединения $\mathrm{SrOs}_{2} \mathrm{O}_{6}$ была рассмотрена химическая реакция

$$
\mathrm{SrO}_{2}+2 \mathrm{OsO}_{2}=\mathrm{SrOs}_{2} \mathrm{O}_{6}
$$

На первом этапе были получены зависимости полной энергии от объема для $\mathrm{SrOs}_{2} \mathrm{O}_{6}, \mathrm{SrO}_{2}$ и $\mathrm{OsO}_{2}$ в результате серии расчетов, в которых объем элементарной ячейки варьировался в пределах $\pm 10 \%$ от среднего значения (рис. 2).

Для оценки значений равновесного объема и энергии зависимости $E(V)$ были аппроксимированы уравнением состояния Берча-Мурнагана 3-го порядка

$$
\begin{aligned}
E(V)= & E_{0}+\frac{9 V_{0} B_{0}}{16}\left(\left(\left(\frac{V_{0}}{V}\right)^{2 / 3}-1\right)^{3} B_{0}^{\prime}\right. \\
& \left.+\left(\left(\frac{V_{0}}{V}\right)^{2 / 3}-1\right)^{2}\left(6-4\left(\frac{V_{0}}{V}\right)^{2 / 3}\right)\right)
\end{aligned}
$$

где $V_{0}$ - объем элементарной ячейки при нормальном давлении, $E_{0}$ - энергия, соответствующая равновесному объему, $B_{0}$ - модуль (коэффициент) всестороннего сжатия и $B_{0}^{\prime}$ производная модуля $B_{0}$ по давлению. Значения величин $E_{0}, V_{0}, B_{0}, B_{0}^{\prime}$, полученные в результате аппроксимации, представлены в табл. 1.

Анализ табл. 1 показывает, что вычисленные значения равновесных объемов $V_{0}$ близки к экспериментальным, так что величина $\left(V_{0}-V_{0}^{\exp }\right) / V_{0}^{\exp }$ составляет $5.2 \%$ для $\mathrm{SrO}_{2}$ [23] и 0.022 для $\mathrm{OsO}_{2}$ [24]. Полученные значения являются типичной оценкой для DFT-расчетов [25].

Термодинамическая стабильность $\mathrm{SrOs}_{2} \mathrm{O}_{6}$ была изучена путем сравнения энтальпий для $\mathrm{SrOs}_{2} \mathrm{O}_{6}$ и
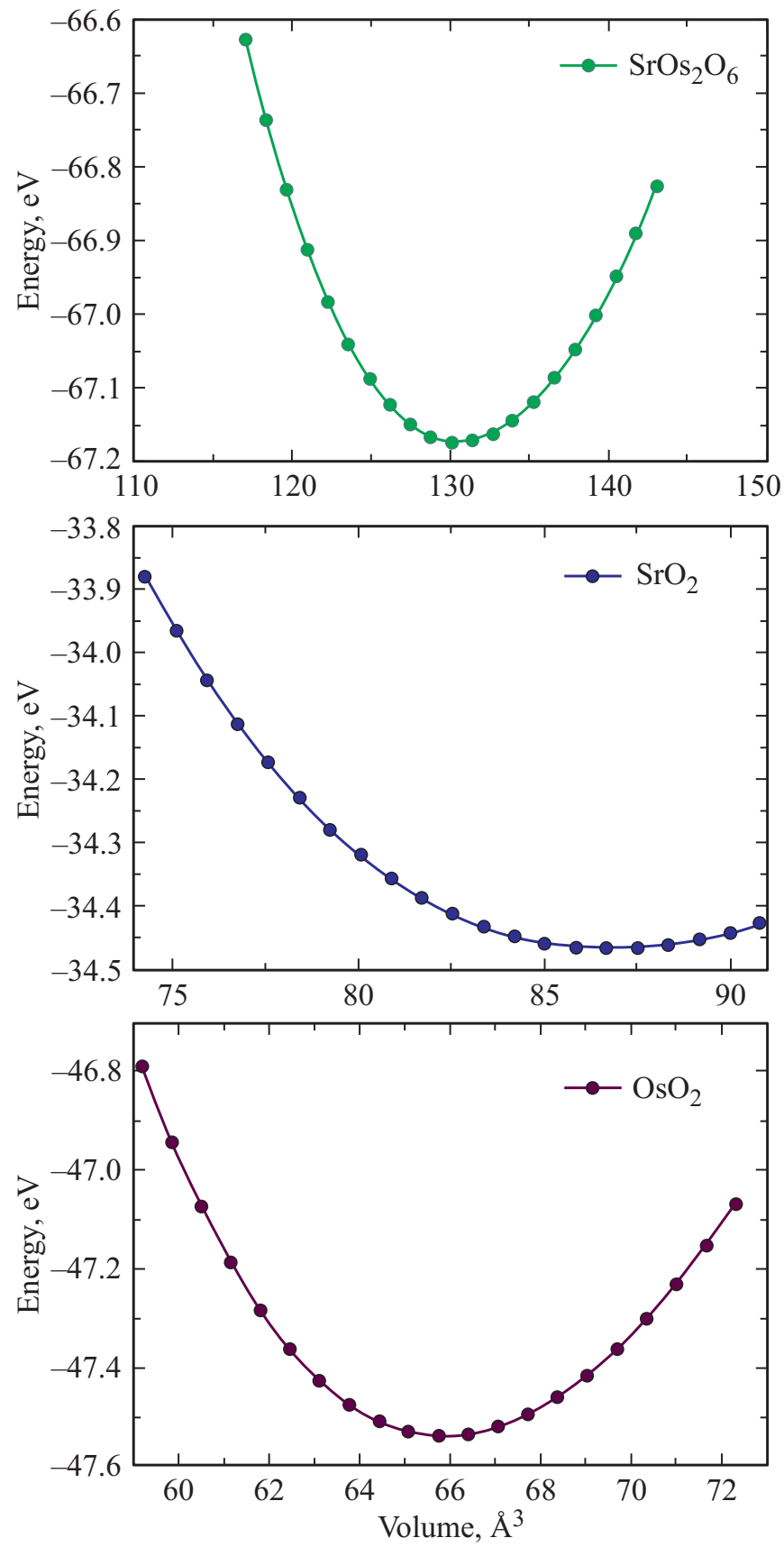

Рис. 2. Зависимости полной энергии от объема элементарной ячейки для $\mathrm{SrOs}_{2} \mathrm{O}_{6}, \mathrm{SrO}_{2}$ и $\mathrm{OsO}_{2}$.

$\mathrm{SrO}_{2}+2 \mathrm{OsO}_{2}$, входящих в реакцию (1), что показано на рис. 3.

Из рис. 3 видно, что соединение $\mathrm{SrOs}_{2} \mathrm{O}_{6}$ остается стабильным при нормальных условиях, что позволяет нам перейти к дальнейшему рассмотрению его кристаллической и электронной структур, а также зонного спектра.

Кристаллическая структура $\mathrm{SrOs}_{2} \mathrm{O}_{6}$ описывается группой симметрии $P-31 \mathrm{~m}$, оптимизированные в DFT параметры решетки и позиции атомов представлены в табл. 2. Атомы Os находятся в вершинах шести- 


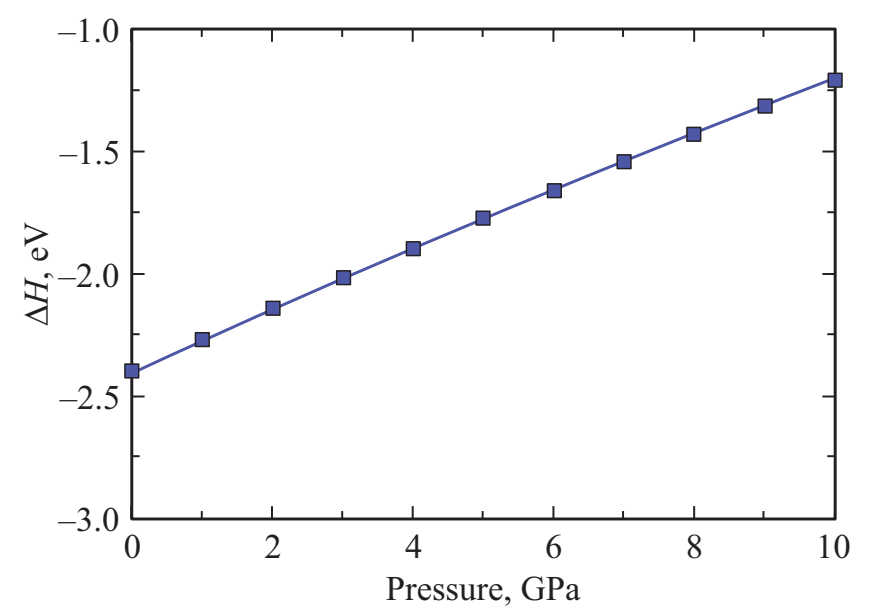

Рис. 3. Зависимость расчетной относительной энтальпии $\Delta H$ $\mathrm{SrOs}_{2} \mathrm{O}_{6}$ по отношению к $\left(\mathrm{SrO}_{2}+2 \mathrm{OsO}_{2}\right)$ от давления.

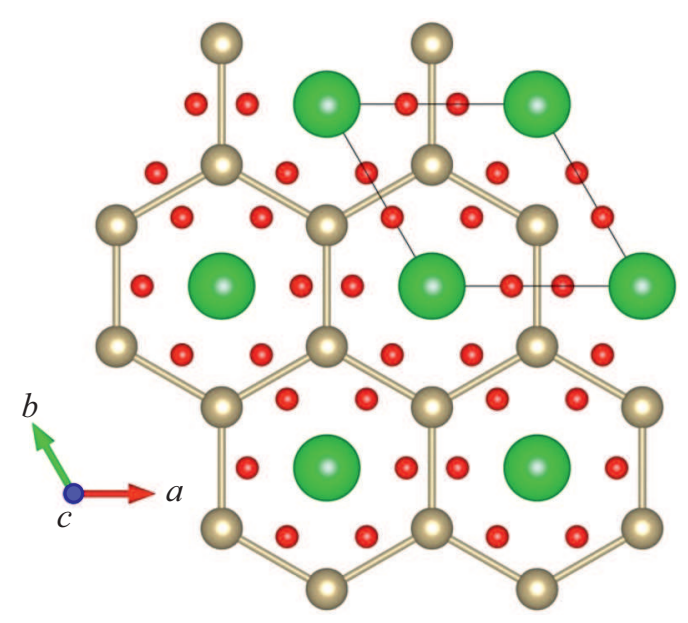

Рис. 4. Проекция кристаллической структуры $\mathrm{SrOs}_{2} \mathrm{O}_{6}$ на плоскость $a b$. Атомы Os представлены средними по размеру (коричневыми) шарами, соединенными линиями, показывающими образование пчелиных сот. Атомы кислорода - маленькие (красные) шары, а $\mathrm{Sr}-$ наибольшие (зеленые) шары в центре сот.

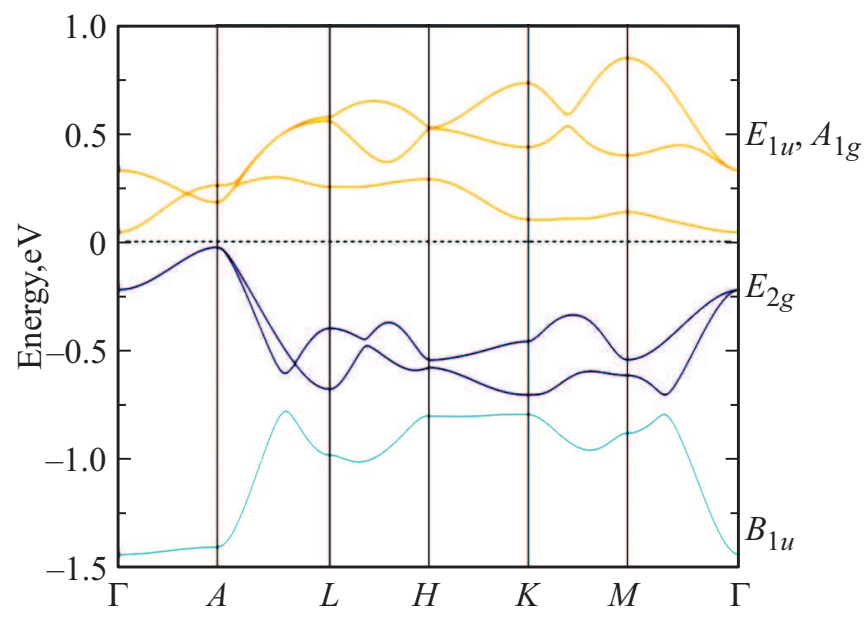

Рис. 5. Зонная структура $\mathrm{SrOs}_{2} \mathrm{O}_{6}$, полученная для немагнитного состояния.
Таблица 1. Параметры уравнения состояния для соединений $\mathrm{SrOs}_{2} \mathrm{O}_{6}, \mathrm{SrO}_{2}, \mathrm{OsO}_{2}$, полученные путем аппроксимации зависимостей $E(V)$ уравнением состояния Берча-Мурнагана 3-го порядка

\begin{tabular}{c|c|c|c}
\hline Параметр & $\mathrm{SrOs}_{2} \mathrm{O}_{6}$ & $\mathrm{SrO}_{2}$ & $\mathrm{OsO}_{2}$ \\
\hline$E_{0}, \mathrm{eV}$ & -67.2 & -34.5 & -47.5 \\
\hline$V_{0}, \AA$ & 130.5 & 86.8 & 65.9 \\
\hline$B_{0}, \mathrm{GPa}$ & 106.4 & 77.3 & 286.3 \\
\hline$B_{0}^{\prime}$ & 3.8 & 4.7 & 4.7
\end{tabular}

Таблица 2. Параметры решетки и координаты атомов для соединения $\mathrm{SrOs}_{2} \mathrm{O}_{6}$

\begin{tabular}{c|c|l}
\hline Симметрия & Параметры решетки & \multicolumn{1}{|c}{ Позиции атомов } \\
\hline$P-31 m$ & $a=5.37152 \AA$ & Sr: $a(0.000,0.000,0.000)$ \\
& $c=5.25716 \AA$ & Os: $d(0.3330 .6670 .500)$ \\
& & O: $k(0.3780 .0000 .301)$
\end{tabular}

угольников, образующих решетку типа пчелиные соты, см. рис. 4. Атомы $\mathrm{Sr}$ и О располагаются между плоскостями, образованными атомами Os. Таким образом соединение $\mathrm{SrOs}_{2} \mathrm{O}_{6}$ изоструктурно соединению $\mathrm{SrRu}_{2} \mathrm{O}_{6}$, и можно ожидать, что его электронный спектр может быть описан концепцией квазимолекулярных орбиталей с центром на шестиугольниках $\mathrm{Os}^{5+}$, предложенной в работе [5], а зонные состояния могут быть классифицированы с помощью неприводимых представлений группы $D_{6 h}$.

На рис. 5 представлена зонная структура, полученная в расчете методом DFT без учета магнитного взаимодействия для соединения $\mathrm{SrOs}_{2} \mathrm{O}_{6}$. Вблизи уровня Ферми находится шесть зон, которые могут быть описаны с помощью квазимолекулярных орбиталей с симметрией $A_{1 g}, E_{1 u}, E_{2 g}, B_{1 u}\left(E_{1 u}\right.$ и $E_{2 g}$ дважды вырождены) [10]. Указанные зоны имеют преимущественно характер Os $t_{2 g}$ (два атома Os в элементарной ячейке). Три из них полностью заполнены $\left(A_{1 g}\right.$ и двукратно вырожденная $\left.E_{1 u}\right)$ и отделены щелью от $E_{2 g}$ и $B_{1 u}$, так же как и в случае $\mathrm{SrRu}_{2} \mathrm{O}_{6}$.

Помимо DFT-расчетов без учета магнитного взаимодействия было проведено моделирование для ферромагнитной и антиферромагнитной (ближайшие соседи антиферромагнитны) конфигурации спинов ионов $\mathrm{Os}^{5+}$. Расчеты показали, что энергия, соответствующая антиферромагнитному состоянию, ниже энергии, соответствующей ферромагнитному состоянию. Разница энергий составляет $\delta E=0.145 \mathrm{eV} /$ f.u. (f.u. - формульная единица). Данный результат свидетельствует о том, что основное антиферромагнитное состояние является более выгодным и именно его появления можно ожидать в низкотемпературной области $\mathrm{SrOs}_{2} \mathrm{O}_{6}$. Рассчитанный из 
первых принципов магнитный момент для антиферромагнитной конфигурации спинов ионов $\mathrm{Os}^{5+}$ оказывается сильно подавленным и равным $1.34 \mu_{\mathrm{B}}$, где $\mu_{\mathrm{B}}-$ магнетон Бора, что может объясняться как существенной гибридизацией с кислородом, так и эффектом образования квазимолекулярных орбиталей.

\section{4. Заключение}

В настоящей работе было проведено моделирование структурных и электронных свойств нового материала - оксида $(\mathrm{V})$ осмия $\mathrm{SrOs}_{2} \mathrm{O}_{6}$ из первых принципов. Показано, что данное соединение является термодинамически стабильным при нормальных условиях. Получены кристаллическая структура $\mathrm{SrOs}_{2} \mathrm{O}_{6}$ и зонный спектр для немагнитного состояния, который описывается концепцией квазимолекулярных орбиталей с центром на шестиугольниках $\mathrm{Os}^{5+}$, при этом зонные состояния могут быть классифицированы с помощью неприводимых представлений группы $D_{6 h}$. Показано, что энергетически выгодным для $\mathrm{SrOs}_{2} \mathrm{O}_{6}$ является антиферромагнитное состояние.

\section{Финансирование работы}

Работа выполнена при поддержке Российского Научного Фонда (проект № 20-62-46047) и Министерства образования и науки РФ (проект № FEUZ-2020-0054). При проведении работ был использован суперкомпьютер „Уран“ ИММ УрО РАН.

\section{Конфликт интересов}

Авторы заявляют об отсутствии конфликта интересов.

\section{Список литературы}

[1] S.M. Winter, A.A. Tsirlin, M. Daghofer, J. van den Brink, Y. Singh, P. Gegenwart, R. Valenti. J. Phys. Condens. Matter 29, 493002 (2017).

[2] H. Takagi, T. Takayama, G. Jackeli, G. Khaliullin, S.E. Nagler. Nature Rev. Phys. 1, 264 (2019).

[3] D.I. Khomskii, S.V. Streltsov. Chem. Rev. 121, 2992 (2021).

[4] S.A.J. Kimber, I.I. Mazin, J. Shen, H.O. Jeschke, S.V. Streltsov, D.N. Argyriou, R. Valenti, D.I. Khomskii. Phys. Rev. B 89, 081408 (2014).

[5] Z.V. Pchelkina, A.L. Pitman, A. Moewes, E.Z. Kurmaev, T.-Y. Tan, D.C. Peets, J.-G. Park, S.V. Streltsov. Phys. Rev. B 91, 115138 (2015).

[6] J. Park, T. Tan, D.T. Adroja, A. Daoud-Aladine, S. Choi, D. Cho, S. Lee, J. Kim, H. Sim, T. Morioka, H. Nojiri, V.V Krishnamurthy, P. Manuel, M.R. Lees, S.V. Streltsov, D.I. Khomskii, J.-G. Park. Sci. Rep. 6, 25238 (2016).

[7] I.I. Mazin, H.O. Jeschke, K. Foyevtsova, R. Valentí, D.I. Khomskii. Phys. Rev. Lett. 109, 197201 (2012).

[8] Z.V. Pchelkina, S.V. Streltsov, I.I. Mazin. Phys. Rev. B 94, 205148 (2016).

[9] S.V. Streltsov. Phys. Met. Metallogr. 119, 1276 (2018).
[10] S. Streltsov, I.I. Mazin, K. Foyevtsova. Phys. Rev. B 92, 134408 (2015).

[11] C.I. Hiley, M.R. Lees, J.M. Fisher, D. Thompsett, S. Agrestini, R.I. Smith, R.I. Walton. Angew. Chem. Int. Ed. 53, 4423 (2014).

[12] W. Tian, C. Svoboda, M. Ochi, M. Matsuda, H.B. Cao, J. Cheng, B.C. Sales, D.G. Mandrus, R. Arita, N. Trivedi, J. Yan. Phys. Rev. B 92, 100404 (2015).

[13] H. Suzuki, H. Gretarsson, H. Ishikawa, K. Ueda, Z. Yang, H. Liu, H. Kim, D. Kukusta, A. Yaresko, M. Minola, J.A. Sears, S. Francoual, H. Wille, J. Nuss, H. Takagi, B.J. Kim, G. Khaliullin, H. Yavaş, B. Keimer. Nature Mater. 18, 563 (2019).

[14] Y.S. Ponosov, E.V Komleva, D.A. Zamyatin, R.I. Walton, S.V Streltsov. Phys. Rev. B 99, 85103 (2019).

[15] B.E. Prasad, S. Kanungo, M. Jansen, A.C. Komarek, B. Yan, P. Manuel, C. Felser. Chem. Eur. J. 23, 1521 (2017).

[16] W. Schnelle, B.E. Prasad, C. Felser, M. Jansen, E.V. Komleva, S.V. Streltsov, I.I. Mazin, D. Khalyavin, P. Manuel, S. Pal, D.V.S. Muthu, A.K. Sood, E.S. Klyushina, B. Lake, J.-C. Orain, H. Luetkens. arXiv:2103.04815

[17] W.A. Harrison. Elementary Electronic Structure. World Scientific, Singapore (1999).

[18] С.В. Стрельцов, Д.И. Хомский. 187, Журн. УФН 1205 (2017).

[19] G. Kresse, J. Hafner. Phys. Rev. B 47558 (1993).

[20] J.P. Perdew, K. Burke, M. Ernzerhof. Phys. Rev. Lett. 77, 3865 (1996).

[21] H. Monkhorst, J. Pack. Phys.Rev. B 13, 5188 (1976).

[22] G. Kresse, D. Joubert. Phys. Rev. B 59, 1758 (1999).

[23] J.D. Bernal, E. Djatlowa, I. Kasarnowsky, S. Reichstein, A.G. Ward. Z. Kristallogr. Cryst. Mater. 92, 344-354 (1935).

[24] Y.C. Venudhar, L. Iyengar, K.V. Krishna Rao. Cryst. Res. Technol. 20, 1393 (1985).

[25] R.M. Martin. Electronic Structure: Basic Theory and Practical Methods. Cambridge University Press, Cambridge (2004).

Редактор Е.Ю. Флегонтова 